\title{
Search for neutrinoless double beta decay with NEMO3 and SuperNEMO
}

\section{Christine MARQUET*}

CENBG University Bordeaux I and CNRS

E-mail: marquet@cenbg.in2p3. fr

The double beta decay experiment NEMO3 has been taking data since February 2003. The aim of this experiment is to search for neutrinoless decay and investigate two neutrino double beta decay in seven different enriched isotopes $\left({ }^{100} \mathrm{Mo},{ }^{82} \mathrm{Se},{ }^{48} \mathrm{Ca},{ }^{96} \mathrm{Zr},{ }^{116} \mathrm{Cd},{ }^{130} \mathrm{Te}\right.$ and $\left.{ }^{150} \mathrm{Nd}\right)$. After analysis of the data corresponding to $4.5 \mathrm{y}$, no evidence for $\beta \beta 0 v$ decay in ${ }^{100} \mathrm{Mo}$ and ${ }^{82} \mathrm{Se}$ was found. The half-life limits at $90 \%$ C.L. are $1.0 \cdot 10^{24} \mathrm{y}$ and $3.2 \cdot 10^{23} \mathrm{y}$, respectively. The measurements of the two-neutrino double beta decays for all the isotopes have also reached the highest precision to date. The SuperNEMO project aims to extend the NEMO technique to a 100$200 \mathrm{~kg}$ isotope experiment with the target half-life sensitivity of 1-2 $10^{26} \mathrm{y}$. The current status of the SuperNEMO R\&D programme is described.

35th International Conference of High Energy Physics

July 22-28, 2010

Paris, France

*Speaker. 


\section{Introduction}

Neutrinoless double beta decay $(\beta \beta 0 v)$ is the most sensitive way to probe the Majorana nature of the neutrinos and to observe the full lepton number violation required by most GUT schemes. It may also determine the abolute mass scale of the neutrinos. The most common mechanism of this decay is the one where a light Majorana neutrino is exchanged but right-handed currents, Majoron emission, R-parity violating SUSY are also possible. The $\beta \beta 0 v$ half-life can be written as :

$$
\left[T_{1 / 2}^{0 v}(A, Z)\right]^{-1}=G_{0 v}\left(Q_{\beta \beta}, Z\right)\left|M_{0 v}(A, Z)\right|^{2}\langle\eta\rangle^{2}
$$

where $G_{0 v}$ is the kinematical factor proportional to the transition energy to the fifth power $Q_{\beta \beta}^{5}, M_{0 v}$ is the nuclear matrix element (NME) and $\langle\eta\rangle$ is a lepton number violating parameter equal to the effective neutrino mass $\left\langle m_{v}\right\rangle$ in the case of a light neutrino exchange.

The $\beta \beta 2 v$ decay process is a rare second order weak interaction process. The measurement of its rate of decay is important since it constitutes the ultimate background in the search for $\beta \beta 0 \mathrm{v}$ decay signal and is a valuable input for the theoretical calculations of the NME.

The objective of the NEMO experiment is the search for the $\beta \beta 0 \mathrm{v}$ decay and an accurate measurement of the $\beta \beta 2 v$ decay. The signature of the $\beta \beta 0 v$ process is the detection of two electrons emitted in coincidence with a total energy sum equal to the transition energy $\mathrm{Q}_{\beta} \beta$. NEMO experiments are based on a "tracko-calo" approach. Such experiments detect not only the total energy deposition but allow also the identification of the particles $\left(\mathrm{e}^{-}, \mathrm{e}^{+}, \alpha, \gamma\right)$ and the measurement of the angular correlations, individual energies and time of flight of emitted electrons. With such information, the NEMO technique provides a way of discriminating the underlying physics mechanism [1] and leads to a very low background experiment.

\section{The NEMO3 experiment}

The NEMO3 detector is located in the LSM underground laboratory shielded from cosmic rays by $1700 \mathrm{~m}$ of rock ( $4800 \mathrm{~m}$ of water equivalent) and has been taking data since February 2003 .

\section{The NEMO3 detector}

The NEMO3 detector is a cylinder made of 20 sectors containing different $\beta \beta$ isotopes (Fig. 1).

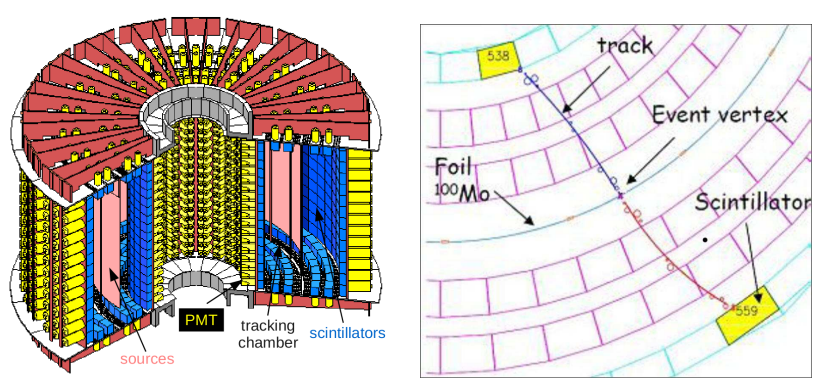

Figure 1: NEMO3 detector and reconstructed $\beta \beta$ event in NEMO3.

Thin source foils $\left(\sim 50 \mathrm{mg} / \mathrm{cm}^{2}\right)$ are located in the middle of the tracking volume surrounded by the calorimeter. They are composed of almost $10 \mathrm{~kg}$ of enriched isotopes listed in Table 1 . The $6.9 \mathrm{~kg}$ of ${ }^{100} \mathrm{Mo}$ and the $0.9 \mathrm{~kg}{ }^{82} \mathrm{Se}$ are used to search for $\beta \beta 0 v$ decay, smaller amounts of ${ }^{130} \mathrm{Te}$, ${ }^{116} \mathrm{Cd},{ }^{150} \mathrm{Nd},{ }^{96} \mathrm{Zr}$ and ${ }^{48} \mathrm{Ca}$ are used to measure $\beta \beta 2 v$ decay and backgrounds. The tracking volume consists of $6180 \mathrm{drift}$ cells operating in Geiger mode. The resolutions on the position in the 
tracker are $0.3 \mathrm{~mm}$ (transverse) and $0.8 \mathrm{~cm}$ (longitudinal). The calorimeter is made of 1940 large blocks of plastic scintillator coupled to low radioactivity 3" or 5" PMTs. The energy resolution of the NEMO3 calorimeter is $14-17 \% / \sqrt{E}(\mathrm{MeV})$ and the time resolution is 250 ps. A $2.5 \mathrm{mT}$ magnetic field is used for charge identification. The detector is shielded from external gamma rays by $18 \mathrm{~cm}$ of low activity iron and against neutrons by $30 \mathrm{~cm}$ of water with boric acid. The events are selected by requiring two reconstructed electron tracks in time starting from a common vertex in the source with a curvature corresponding to a negative charge (Fig. 1). Two phases of data have to be considered: high radon and low radon phases, because of the radon-free air facility installation in the LSM in 2004 flushing a tight tent around the detector [2] .

\section{NEMO3 results}

The NEMO3 detector is able to measure all its background components using event topologies. A background model has been built detailed in [3]. The measurements of the $\beta \beta 2 v$ decay half-lives were performed for the seven isotopes of NEMO3 (Table 1) with unprecedented precision.

\begin{tabular}{|c|c|c|c|c|c|}
\hline Isotope & Mass [g] & $Q_{\beta \beta}[\mathrm{keV}]$ & Sig/Bkg & $T_{1 / 2}\left[10^{19}\right.$ years $]$ & $M^{2 v}$ \\
\hline${ }^{100} \mathrm{Mo}$ & 6914 & 3034 & 76 & $0.717 \pm 0.001$ (stat) \pm 0.054 (syst) & $0.126 \pm 0.006$ \\
${ }^{82} \mathrm{Se}$ & 932 & 2995 & 4 & $9.6 \pm 0.1$ (stat) \pm 1.0 (syst) & $0.049 \pm 0.004$ \\
${ }^{130} \mathrm{Te}$ & 454 & 2529 & 0.25 & $70_{-8}^{+10}$ (stat) ${ }_{-9}^{+10}$ (syst) & $0.017 \pm 0.003$ \\
${ }^{116} \mathrm{Cd}$ & 405 & 2805 & 10.3 & $2.88 \pm 0.04$ (stat) \pm 0.16 (syst) & $0.069 \pm 0.003$ \\
${ }^{150} \mathrm{Nd}$ & 37.0 & 3368 & 2.8 & $0.920 \pm 0.025$ (stat) \pm 0.063 (syst) & $0.030 \pm 0.002[4]$ \\
${ }^{96} \mathrm{Zr}$ & 9.4 & 3350 & 1.0 & $2.35 \pm 0.14$ (stat) \pm 0.16 (syst) & $0.049 \pm 0.002[5]$ \\
${ }^{48} \mathrm{Ca}$ & 6.99 & 4274 & 6.8 & $4.4_{-0.4}^{+0.5}$ (stat) \pm 0.4 (syst) & $0.024 \pm 0.002$ \\
\hline
\end{tabular}

Table 1: NEMO3 results of the $\beta \beta 2 v$ half-life measurements.

The most precise measurement is for the main isotope ${ }^{100}$ Mo because of the high mass and the high signal to background ratio due to a long $T_{1 / 2}^{2 v}$ half-life. With this result we can appreciate the accuracy of the NEMO3 technique by looking at the distribution of the total and individual energies and the angle between the two electrons (Fig. 2). A measurement of the two-neutrino double beta decay to excited states has also been obtained for the $0^{+} \rightarrow 0_{1}^{+}$transition: $T_{1 / 2}^{2 v}=$ $5.7_{-0.9}^{+1.3}$ stat. \pm 0.8 syst. $10^{20} \mathrm{yr}$ and a $90 \%$ CL limit for the $0^{+} \rightarrow 2_{1}^{+}$decay: $T_{1 / 2}^{2 v}>1.110^{21} \mathrm{yr}$.
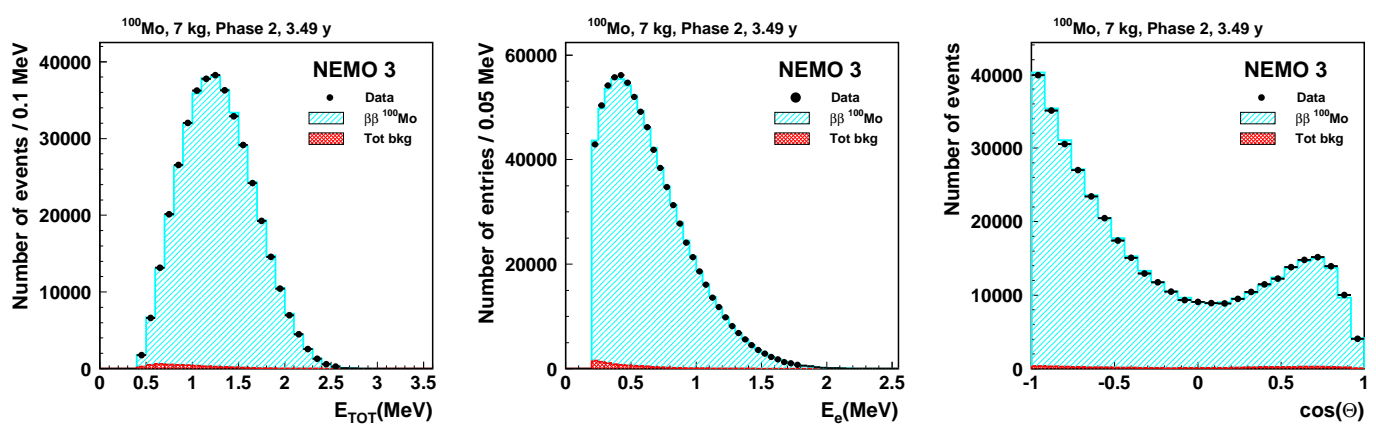

Figure 2: Total energy, individual energy and angular distributions of the ${ }^{100} \mathrm{Mo} \beta \beta 2 v$ events in the NEMO3 experiment for the low radon data phase (3.49 years).

The distribution of the two electron energy sum around $Q_{\beta \beta}$ of ${ }^{100} \mathrm{Mo}$ and ${ }^{82} \mathrm{Se}$ was used to search for the $\beta \beta 0 v$ decay. No evidence for $\beta \beta 0 v$ signal has been observed, leading to a 90\% CL limit on the half-lives set to: $T_{1 / 2}^{0 v}>1.010^{24} \mathrm{yr}$ for ${ }^{100} \mathrm{Mo}$ and $T_{1 / 2}^{0 v}>3.210^{23} \mathrm{yr}$ for ${ }^{82} \mathrm{Se}$. The corresponding limits on the effective Majorana neutrino mass are respectively $\left\langle m_{v}\right\rangle<0.47-0.96 \mathrm{eV}$ and 
$\left\langle m_{v}\right\rangle<0.94-2.5 \mathrm{eV}$, according to the NME calculations [6] [7] [8].Other mechanisms of $\beta \beta 0 v$ decay have been investigated and limits have been set for decays to excited states: $T_{1 / 2}^{0 v}\left(0^{+} \rightarrow\right.$ $\left.0_{1}^{+}\right)>8.910^{22} \mathrm{yr}$ and $T_{1 / 2}^{0 v}\left(0^{+} \rightarrow 2_{1}^{+}\right)>1.610^{23} \mathrm{yr}$, for $\mathrm{V}+\mathrm{A}$ currents: $T_{1 / 2}^{0 v}>5.410^{23} \mathrm{yr}$ and for Majoron emission: $T_{1 / 2}^{0 v}>2.710^{22} \mathrm{yr}$.

\section{The SuperNEMO project}

The SuperNEMO project aims to extend and improve the successful NEMO3 technology. It will extrapolate NEMO3 by two orders of magnitude on the $\beta \beta 0 \mathrm{v}$ half-lives sensitivity, studying about $100 \mathrm{~kg}$ of $\beta \beta$ isotopes and improving several parameters shown in Table 2. The SuperNEMO design envisages twenty identical modules, each housing $5 \mathrm{~kg}$ of isotopes in a thin foil sandwiched between two planar tracking chambers and closed by calorimeter walls (Fig. 3).

\begin{tabular}{rrr}
\hline Experiment & NEMO3 & SuperNEMO \\
\hline Choice of isotope & ${ }^{100} \mathrm{Mo}$ & ${ }^{82} \mathrm{Se}\left(\right.$ or $\left.{ }^{150} \mathrm{Nd} \mathrm{or}{ }^{48} \mathrm{Ca}\right)$ \\
Isotop mass & $7 \mathrm{~kg}$ & $100-200 \mathrm{~kg}$ \\
Internal contaminations & ${ }^{208} \mathrm{Tl} \sim 100 \mu \mathrm{Bq} / \mathrm{kg}$ & ${ }^{208} \mathrm{Tl}<2 \mu \mathrm{Bq} / \mathrm{kg}$ \\
${ }^{208} \mathrm{Tl}$ and ${ }^{214} \mathrm{Bi}$ in the foil & ${ }^{214} \mathrm{Bi}<300 \mu \mathrm{Bq} / \mathrm{kg}$ & ${ }^{208} \mathrm{Tl}<10 \mu \mathrm{Bq} / \mathrm{kg}$ \\
Energy resolution FWHM (calorimeter) & $8 \%$ at $3 \mathrm{MeV}$ & $4 \%$ at $3 \mathrm{MeV}$ \\
Sensitivity & $T_{1 / 2}(\beta \beta 0 \mathrm{v})>2.10^{24} \mathrm{y}$ & $T_{1 / 2}(\beta \beta 0 \mathrm{v})>10^{26} \mathrm{y}$ \\
& $<m_{v}><0.3-0.9 \mathrm{eV}$ & $<m_{v}><40-110 \mathrm{meV}$ \\
\hline
\end{tabular}

Table 2: Characteristics of the NEMO3 experiment and of the SuperNEMO project.

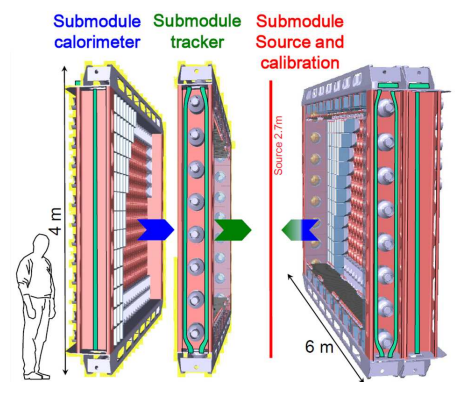

Figure 3: A SuperNEMO module.

\section{SuperNEMO design study}

The R\&D programme carried out since 2006 has addressed the main challenges : improvement of the calorimeter energy resolution, radiopurity of the source foils and optimisation of the tracker. - $\beta \beta$ Source: the feasability of enrichement by centrifugation for $100 \mathrm{~kg}$ and purification methods have been evaluated. The measurement of the ultra-low levels of ${ }^{208} \mathrm{Tl}$ and ${ }^{214} \mathrm{Bi}$ contaminations in the source foils (Table 2) requires a dedicated tool. The "BiPo" detector has been developed by exploiting the beta and delayed alpha decays occuring in the natural radioactive chains of ${ }^{208} \mathrm{Tl}$ and ${ }^{214} \mathrm{Bi}$. The target sensitivity of $10 \mu \mathrm{Bq} / \mathrm{kg}$ has been reached with the BiPo1 prototype [9].

- Calorimeter: the energy resolution is a key factor in discriminating a $\beta \beta 0 v$ signal from $\beta \beta 2 v$ background. A large number of studies have been carried out to investigate the material, size, shape and coating of the calorimeter blocks as well as performance and intrinsic radioactivity of the PMTs. The feasibility to reach the required energy resolution of $8 \% / \sqrt{E}(\mathrm{MeV})$ with a large $\left(26 \times 26 \times 15 \mathrm{~cm}^{3}\right)$ block has been experimentally demonstrated with PVT-based scintillators coupled to a low radioactive high-QE 8" R5912MOD Hamamatsu PMT. 
- Tracker: the tracker will consist of drift cells operating in Geiger mode. Tests on small prototypes have been worked out to optimize the choice of the wire material, size, diameter, position and readout. Measurements of cosmic ray tracks in a 90-cells prototype have demonstrated the required resolutions $(0.7 \mathrm{~mm}$ radial and $1 \mathrm{~cm}$ longitudinal). In parallel, an automated wiring robot has been developed for mass production of the cells (400.000 in SuperNEMO).

\section{Demonstrator and schedule}

The SuperNEMO collaboration has started the construction of the first module, the demonstrator. Its main goals are to demonstrate feasibility of mass production, to measure backgrounds especially from radon emanation, to finalise the detector design and to produce a competitive physics result. To accomplish the latter goal on a competitive time scale the demonstrator module will house $7 \mathrm{~kg}$ of the ${ }^{82} \mathrm{Se}$ isotope. The construction and commissioning of the demonstrator will be completed in 2013 with data taking expected to start mid-2013. The sensitivity of the demonstrator after $17 \mathrm{~kg} . \mathrm{yr}$ of exposure is $6.510^{24} \mathrm{yr}(90 \% \mathrm{CL})$. This sensitivity will allow an experimental verification of a recent claim of evidence for $\beta \beta 0 v$ [10]. The full detector construction is expected to start in 2014 (in parallel with the demonstrator running). The $500 \mathrm{~kg}$.yr exposure will be reached in 2019, pushing the sensitivity to the effective Majorana neutrino mass down to 50-100 meV.

\section{Conclusions}

Neutrinoless double beta decay is a powerful way of addressing lepton number violation and the nature and absolute mass scale of neutrinos. The NEMO experiment offers a unique "trackocalo" approach to the detection of the process, in which the topology of the decay is fully reconstructed. Apart from producing a clear signature of the process, these detectors offer a superior background rejection capability and allow the underlying physics mechanism of $\beta \beta 0 v$ to be disentangled. The NEMO3 detector gave the best $\beta \beta 2 v$ half-lives measurements for 7 isotopes and competitive limits on $\beta \beta 0 v$ decays. The successful technology will be extrapolated to SuperNEMO whose R\&D reached the required performances. Construction and commissioning of the first module (demonstrator) will be completed in 2013 for a possible full detector construction starting in 2014 reaching its sensitivity of $10^{26} \mathrm{yr}$ on $\beta \beta 0 v$ decay of ${ }^{82} \mathrm{Se}$ in 2019.

\section{References}

[1] R. Arnold et al, Eur. Phys. J. C 70 (2010) 927.

[2] R. Arnold et al, Nucl. Instrum. Methods A 536 (2005) 79.

[3] J. Argyriades et al, Nucl. Instrum. Methods A 606 (2009) 449.

[4] J. Argyriades et al, Phys. Rev. C 80 (2009) 032501(R)

[5] J. Argyriades et al, Nucl. Phys. A 847 (2010) 168

[6] E. Caurier et al, Phys. Rev. Lett. 100 (2008) 052503

[7] J.Suhonen et al, Int. J. Mod. Phys. E 17 (2008) 1.

[8] F. Šimkovich et al, Phys. Rev. C 77 (2008) 045503.

[9] J. Argyriades et al, Nucl. Instrum. Methods A 622 (2010) 120 .

[10] H.V. Klapdor-Kleingrothaus and I.V. Krivosheina, et al, Mod. Phys. Lett. A 21 (2006) 1547. 